\section{Pulmonary disease following intravesical BCG treatment}

\author{
S Kesten, L Title, B Mullen, R Grossman
}

\begin{abstract}
The potentially toxic nature of intravesical BCG is illustrated by two patients who developed profound systemic illness with fever, rash, basal crackles, and bilateral shadowing on the chest radiograph after treatment.
\end{abstract}

Bacillus Calmette-Guérin (BCG) is an attenuated strain of Mycobacterium bovis that has been used in the treatment of malignant disease for over 20 years and for the treatment of bladder cancer since $1976 .^{1}$ Major complications of this treatment are infrequent. ${ }^{2}$ We report two cases of systemic illness with pulmonary manifestations after treatment with intravesical BCG.

\footnotetext{
Mount Sinai Hospital, Toronto, Canada S Kesten, L Title, B Mullen, R Grossman

Address for reprint requests: Dr S Kesten,

Toronto Western Hospital

(Edith Cavell Wing),

399 Bathurst Street,

Toronto, Ontario, Canada

M5T 2S8.

Accepted 25 April 1990
}

CASE 1

A 67 year old man with in situ transitional cell carcinoma of the bladder was treated with suspensions of $120 \mathrm{mg}$ of BCG in $60 \mathrm{ml}$ of saline instilled into the bladder at weekly intervals. The initial full blood count, liver enzymes, and chest radiograph were normal.

\section{Case reports}

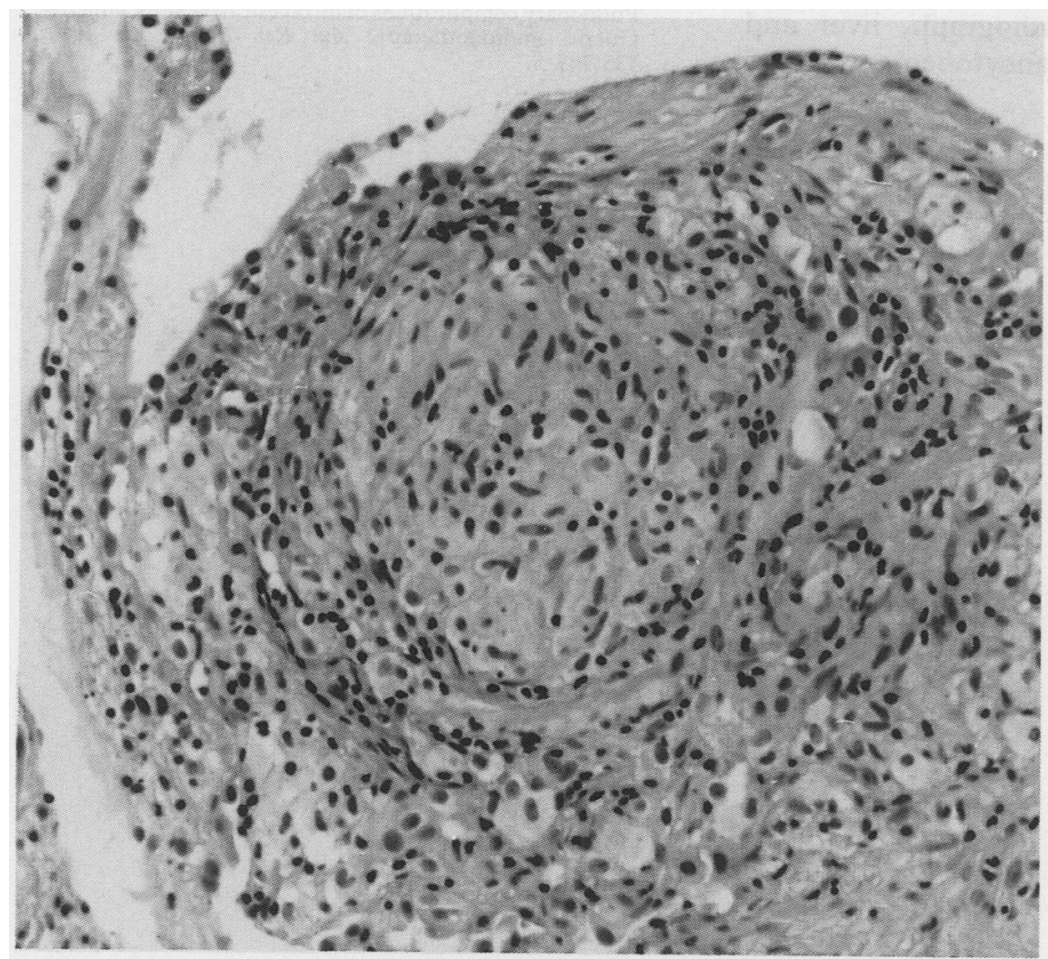

Section of an open lung biopsy specimen showing non-caseating granuloma and alveolar lining cell hyperplasia with mild chronic inflammatory cell infiltration in the adjacent pulmonary parenchyma. (Haematoxylin and eosin.)
Immediately after the fourth treatment he developed fever and urinary symptoms. Urine analysis showed numerous white cells and bacteria but cultures were negative. One month later he complained of intermittent fever, and a $4.5 \mathrm{~kg}$ weight loss.

Fever, bilateral basal inspiratory crackles, numerous small $(<5 \mathrm{~mm})$ erythematous macules with haemorrhagic centres on his back and a few petechial lesions on his shins were noted. Pancytopenia was present with a haemoglobin concentration of $11.5 \mathrm{~g} / \mathrm{dl}$, white cell count of $3.8 \times 10^{9} / 1$, and platelet count of $14 \times 10^{9} / 1$. Alkaline phosphatase, aspartate transaminase, alanine transaminase and $\gamma$ glutamyl transferase were two to three times normal. Diffuse bilateral microreticulonodular shadowing was noted on his chest radiograph. A gallium scan showed a $4+$ diffuse lung uptake bilaterally with an enlarged spleen indicated by increased uptake. Bronchoalveolar lavage revealed $30 \%$ neutrophils, $15 \%$ lymphocytes, and $55 \%$ alveolar macrophages. Blood, urine, sputum, lavage fluid and bone marrow cultures were negative. Bone marrow biopsy showed non-caseating granulomas.

The patient was diagnosed as having disseminated BCG and was started on isoniazid $300 \mathrm{mg}$ and rifampin $600 \mathrm{mg}$ daily on his sixth day in hospital. His fever resolved and he was clinically improved by the eighth day of treatment (about six weeks after his last course of intravesical BCG). After one month of treatment his haemoglobin was $12.7 \mathrm{~g} / \mathrm{dl}$ and the white cell count, platelet count, liver enzymes, and chest radiograph had returned to normal.

CASE 2

A 75 year old man was treated with intravesical BCG $(120 \mathrm{mg}$ of BCG in $60 \mathrm{ml}$ of saline) for transitional cell carcinoma of the bladder. After his third monthly treatment he presented with a seven day history of cough and fever and increasing dyspnoea for one day. He was febrile and he had bilateral basal inspiratory crackles and palpable purpuric skin lesions 3-4 $\mathrm{mm}$ in diameter over the thorax. A full blood count showed mild leucocytosis. Alkaline phosphatase activities were mildly increased. A chest radiograph showed bilateral interstitial infiltrates. Granulomas were seen on transbronchial lung biopsy. Skin biopsy showed erythema multiforme; neither granulomas nor acid fast bacilli were seen.

Intensive care was needed because of hypoxaemic respiratory failure five days after admission to hospital. Isoniazid $300 \mathrm{mg}$ daily, rifampin $600 \mathrm{mg}$ daily, and prednisone $60 \mathrm{mg}$ daily were started on the day of his transfer. On the 14th day an open lung biopsy was performed because of increasing hypoxaemia and progressive radiographic infiltrates. Diffuse alveolar damage (acute and organising) and granulomatous inflammation were noted (figure). No acid fast bacilli were seen and cultures were negative for mycobacteria. Viral studies, blood cultures, and serological tests for atypical organisms gave negative results. 
Isoniazid was replaced with ethambutol $1 \mathrm{~g}$ daily because of increased liver enzyme activities. The patient required a prolonged course of mechanical ventilation, which was complicated by a pneumothorax. Clinical improvement in his respiratory condition did not occur until about seven weeks after the start of antituberculous treatment. The chest radiograph and liver enzyme activities gradually returned to normal. The patient was discharged home about 19 weeks after admission to hospital.

\section{Discussion}

Intravesical BCG has been used successfully in the treatment of bladder tumours since $1976,{ }^{13}$ skin test reactivity being useful in predicting a response to treatment. ${ }^{4}$ Intravesical BCG is regarded as a fairly benign treatment, which is well tolerated with infrequent major side effects. Minor complications such as irritative bladder symptoms and fever are most frequent and usually resolve without specific treatment. ${ }^{2}$ In 1278 treated patients cystitis occurred in $91 \%$, fever of over $103^{\circ} \mathrm{F}$ in $3.9 \%$, granulomatous prostatitis in $1.3 \%$, pneumonitis or hepatitis in $0.9 \%$, rash in $0.4 \%$, epididymo-orchitis in $0.2 \%$, hypotension in $0.1 \%$, and cytopenia in $0.1 \%{ }^{2}$ Fever usually occurred after at least six treatments. Two cases of granulomatous hepatitis and localised pneumonitis following intravesical BCG occurred in 91 patients. One patient also had a mild leucopenia $(3.7 \times$ $\left.10^{9} / 1\right)$. Recently three cases of bilateral interstitial pneumonitis secondary to intravesicular BCG without evidence of granulomatous inflammation of the lung were reported. ${ }^{6}$

The first case in this report, with a miliary pattern on the chest radiograph, liver and spleen disease, and pancytopenia with a granulomatous bone marrow, showed more widespread dissemination than previously reported from intravesical BCG. The pattern of granulomatous inflammation suggests haematogenous spread, though a hypersensitivity reaction cannot be excluded. ${ }^{6}$ Our second case shows for the first time granulomatous inflammation of the lung with diffuse lung injury, in keeping with the adult respiratory distress syndrome. As mycobacteria were not identified either by acid fast stains or cultures, we cannot firmly determine the mechanism of this process.

Although infrequent, the cases presented and others that have been reported indicate that intravesical BCG may result in appreciable toxicity. Such complications appear to be responsive to standard antituberculous treatment with or without the addition of corticosteroids. ${ }^{256}$

It has been proposed that severe systemic complications may be prevented by prophylactic isoniazid given for three days with each BCG treatment. ${ }^{5}$ This hypothesis has not been formally tested and it is not known whether prophylactic isoniazid might interfere with the antitumour effect of intravesical BCG.

1 Morales A, Eidinger D, Bruce AW. Intra-cavitary bacillus Calmette-Guerin in the treatment of superficial bladder Calmette-Guerin in the treatmen

2 Lamm DL, Stogdill VD, Stogdill BJ, Crispen RG. Complications of bacillus Calmette-Guerin immunotherapy in 1,278 patients with bladder cancer. J Urol 1985; 135:272-4.

3 Brosmoan SA. Experience with bacillus Calmette-Guérin in patients with superficial bladder carcinoma. $J$ Urol 1982; 128:27-9.

4 Lamm DL. Bacillus Calmette-Guérin immunotherapy for bladder cancer. $J$ Urol 1985;134:40-6.

5 Steg A, Sicard D, Leleu C, Debre B, Boccon-Gibod L. Systemic complications of intravesical BCG therapy for bladder cancer. Lancet 1985;ii:899.

6 Israel-Biet D, Venet A, Sandron D, Ziza JM, Chretien J. Pulmonary complications of intravesical bacille CalmetteGuérin immunotherapy. Am Rev Respir Dis 1987; 135:763-5. 